\title{
Analysis of Discrete and Hybrid Stochastic Systems by Nonlinear Contraction Theory
}

\author{
Quang-Cuong Pham \\ Laboratoire de Physiologie de la Perception et de l'Action \\ Collège de France - CNRS \\ Paris, France \\ cuong.pham@normalesup.org
}

\begin{abstract}
We investigate the stability properties of discrete and hybrid stochastic nonlinear dynamical systems. More precisely, we extend the stochastic contraction theorems (which were formulated for continuous systems) to the case of discrete and hybrid resetting systems. In particular, we show that the mean square distance between any two trajectories of a discrete (or hybrid resetting) contracting stochastic system is upper-bounded by a constant after exponential transients. Using these results, we study the synchronization of noisy nonlinear oscillators coupled by discrete noisy interactions.
\end{abstract}

\section{INTRODUCTION}

Contraction theory is a set of relatively recent tools that provide a systematic approach to the stability analysis of a large class of nonlinear dynamical systems [1], [2], [3], [4]. A nonlinear nonautonomous system $\dot{\mathbf{x}}=\mathbf{f}(\mathbf{x}, t)$ is contracting if the symmetric part of the Jacobian matrix of $\mathbf{f}$ is uniformly negative definite in some metric. Using elementary fluid dynamics techniques, it can be shown that contracting systems are incrementally stable, that is, any two system trajectories exponentially converge to each other [1].

From a practical viewpoint, contraction theory has been successfully applied to a number of important problems, such as mechanical observers and controllers design [5], chemical processes control [6], synchronization analysis [2], [7] or biological systems modelling [8].

Recently, contraction analysis has been extended to the case of stochastic dynamical systems governed by Itô differential equations [4]. In parallel, hybrid versions of contraction theory have also been developped [3]. A hybrid system is characterized by a continuous evolution of the system's state, and intermittent discrete transitions. Such systems are pervasive in both artificial (e.g. analog physical processes controlled by digital devices) and natural (e.g. spiking neurons with subthreshold dynamics) environments.

This paper benefits from these recent developments, and provides an exponential stability result for discrete and hybrid systems governed by stochastic difference and differential equations. More precisely, we prove in section [I] and [II] that the mean square distance between any two trajectories of a discrete (respectively hybrid resetting) stochastic contracting system is upper-bounded by a constant after exponential transients. This bound can be expressed as function of the noise intensities and the contraction rates of the noise-free systems. In section IV we briefly discuss a number of theoretical issues regarding our analysis. In section $\mathrm{V}$, we study, using the previously developped tools, the synchronization of noisy nonlinear oscillators that interact by discrete noisy couplings. Finally, some future directions of research are indicated in section VI.

Notations The symmetric part of a matrix $\mathbf{A}$ is defined as $\mathbf{A}_{s}=\frac{1}{2}\left(\mathbf{A}+\mathbf{A}^{T}\right)$. For a symmetric matrix $\mathbf{A}, \lambda_{\min }(\mathbf{A})$ and $\lambda_{\max }(\mathbf{A})$ denote respectively the smallest and the largest eigenvalue of A. A set of symmetric matrices $\left(\mathbf{A}_{i}\right)_{i \in I}$ is uniformly positive definite if $\exists \alpha>0, \forall i \in I, \lambda_{\min }\left(\mathbf{A}_{i}\right) \geq \alpha$. Finally, for a process $\mathbf{x}(t)$, we note $\mathbb{E}_{\mathbf{x}}(\cdot)=\mathbb{E}(\cdot \mid \mathbf{x}(0)=\mathbf{x})$.

\section{DISCRETE SYSTEMS}

We first prove a lemma that makes explicit the initial "discrete contraction" proof (see section 5 of [1]). Note that a similar proof for continuous systems can be found in [9].

Lemma 1 (and definition): Consider two metrics $\mathbf{M}_{i}=$ $\boldsymbol{\Theta}_{i}^{T} \boldsymbol{\Theta}_{i}$ defined over $\mathbb{R}^{n_{i}}(i=1,2)$ and a smooth function $\mathbf{f}: \mathbb{R}^{n_{1}} \rightarrow \mathbb{R}^{n_{2}}$. The generalized Jacobian of $\mathbf{f}$ in the metrics $\left(\mathbf{M}_{1}, \mathbf{M}_{2}\right)$ is defined by

$$
\mathbf{F}=\boldsymbol{\Theta}_{2} \frac{\partial \mathbf{f}}{\partial \mathbf{x}} \Theta_{1}^{-1}
$$

Assume now that $\mathbf{f}$ is contracting in the metrics $\left(\mathbf{M}_{1}, \mathbf{M}_{2}\right)$ with rate $\beta(0<\beta<1)$, i.e.

$$
\forall \mathbf{x} \in \mathbb{R}^{n_{1}} \quad \lambda_{\max }\left(\mathbf{F}(\mathbf{x})^{T} \mathbf{F}(\mathbf{x})\right) \leq \beta
$$

Then for all $\mathbf{u}, \mathbf{v} \in \mathbb{R}^{n}$, one has

$$
d_{\mathbf{M}_{2}}(\mathbf{f}(\mathbf{u}), \mathbf{f}(\mathbf{v}))^{2} \leq \beta d_{\mathbf{M}_{1}}(\mathbf{u}, \mathbf{v})^{2}
$$

where $d_{\mathbf{M}}$ denotes the distance associated with the metric $\mathbf{M}$ (the distance between two points is defined by the infimum of the lengths in the metric $\mathbf{M}$ of all continuously differentiable curves connecting these points).

Proof Consider a $\mathrm{C}^{1}$ curve $\gamma:[0,1] \rightarrow \mathbb{R}^{n_{1}}$ that connects $\mathbf{u}$ and $\mathbf{v}$ (i.e. $\gamma(0)=\mathbf{u}$ and $\gamma(1)=\mathbf{v}$ ). The $\mathbf{M}_{1}$-length of such a curve is given by

$$
L_{\mathbf{M}_{1}}(\gamma)=\int_{0}^{1} \sqrt{\left(\frac{\partial \gamma}{\partial u}(u)\right)^{T} \mathbf{M}_{1}\left(\frac{\partial \gamma}{\partial u}(u)\right)} d u
$$


Since $\mathbf{f}$ is a smooth function, $\mathbf{f}(\gamma)$ is also a $\mathrm{C}^{1}$ curve, with

$$
L_{\mathbf{M}_{2}}(\mathbf{f}(\gamma))=\int_{0}^{1} \sqrt{\left(\frac{\partial \mathbf{f}(\gamma)}{\partial u}(u)\right)^{T} \mathbf{M}_{2}\left(\frac{\partial \mathbf{f}(\gamma)}{\partial u}(u)\right)} d u
$$

The chain rule next implies that

which leads to

$$
\frac{\partial \mathbf{f}(\gamma)}{\partial u}(u)=\frac{\partial \mathbf{f}}{\partial \mathbf{x}} \frac{\partial \gamma}{\partial u}(u)
$$

$$
\begin{aligned}
L_{\mathbf{M}_{2}}(\mathbf{f}(\gamma)) & =\int_{0}^{1} \sqrt{\left(\frac{\partial \frac{\gamma}{\partial u}^{T}}{\partial \mathbf{x}}{ }^{T} \boldsymbol{\Theta}_{2}^{T} \boldsymbol{\Theta}_{2} \frac{\partial \mathbf{f}}{\partial \mathbf{x}} \frac{\partial \gamma}{\partial u}\right)} d u \\
& =\int_{0}^{1} \sqrt{\left(\frac{\partial \gamma}{\partial u} \boldsymbol{\Theta}_{1}^{T}\right) \mathbf{F}^{T} \mathbf{F}\left(\boldsymbol{\Theta}_{1} \frac{\partial \gamma}{\partial u}\right)} d u \\
& \leq \int_{0}^{1} \sqrt{\beta\left(\frac{\partial \gamma}{\partial u} \boldsymbol{\Theta}_{1}^{T} \boldsymbol{\Theta}_{1} \frac{\partial \gamma}{\partial u}\right)} d u \\
& =\sqrt{\beta} L_{\mathbf{M}_{1}}(\gamma)
\end{aligned}
$$

Choose now a sequence of curves $\left(\gamma_{n}\right)_{n \in \mathbb{N}}$ such that $\lim _{n \rightarrow \infty} L_{\mathbf{M}_{1}}\left(\gamma_{n}\right)=d_{\mathbf{M}_{1}}(u, v)$. From (1), one has $\forall n \in$ $\mathbb{N}, L_{\mathbf{M}_{2}}\left(\mathbf{f}\left(\gamma_{n}\right)\right) \leq \sqrt{\beta} L_{\mathbf{M}_{1}}\left(\gamma_{n}\right)$. By definition of distance, one then has $\forall n \in \mathbb{N}, d_{\mathbf{M}_{2}}(\mathbf{f}(u), \mathbf{f}(v)) \leq \sqrt{\beta} L_{\mathbf{M}_{1}}\left(\gamma_{n}\right)$. Finally, by letting $n$ go to infinity in the last inequality, one obtains the desired result.

Theorem 1 (Discrete stochastic contraction): Consider the stochastic difference equation

$$
\mathbf{a}_{k+1}=\mathbf{f}\left(\mathbf{a}_{k}, k\right)+\sigma\left(\mathbf{a}_{k}, k\right) w_{k+1}
$$

where $\mathbf{f}$ is a $\mathbb{R}^{n} \times \mathbb{N} \rightarrow \mathbb{R}^{n}$ function, $\sigma$ is a $\mathbb{R}^{n} \times \mathbb{N} \rightarrow \mathbb{R}^{n d}$ matrix-valued function and $\left\{w_{k}, k=1,2, \ldots\right\}$ is a sequence of independent $d$-dimensional Gaussian noise vectors, with $w_{k} \sim$ $\mathscr{N}\left(\mathbf{0}, \mathbf{Q}_{k}\right)$.

Assume that the system verifies the following two hypotheses

(H1) the dynamics $\mathbf{f}(\mathbf{a}, k)$ is contracting in the metrics $\left(\mathbf{M}_{k}, \mathbf{M}_{k+1}\right)$, with contraction rate $\beta(0<\beta<$ $1)$, and the metrics $\left(\mathbf{M}_{k}\right)_{k \in \mathbb{N}}$ are uniformly positive definite.

(H2) the impact of noise is uniformly upper-bounded by a constant $\sqrt{C}$ in the metrics $\mathbf{M}_{k}$

$$
\forall \mathbf{a}, k \quad d_{\mathbf{M}_{k}}\left(\mathbf{f}(\mathbf{a}, k), \mathbf{f}(\mathbf{a}, k)+\sigma(\mathbf{a}, k) w_{k}\right) \leq \sqrt{C}
$$

Let $\mathbf{a}_{k}$ and $\mathbf{b}_{k}$ be two trajectories whose initial conditions are given by a probability distribution $p\left(\mathbf{x}_{0}\right)=p\left(\mathbf{a}_{0}, \mathbf{b}_{0}\right)$. Then for all $k \geq 0$

$$
\begin{array}{r}
\mathbb{E}\left(d_{\mathbf{M}_{k}}\left(\mathbf{a}_{k}, \mathbf{b}_{k}\right)\right) \leq \frac{2 \sqrt{C}}{1-\sqrt{\beta}}+ \\
\sqrt{\beta}^{k} \int\left[d_{\mathbf{M}_{0}}(\mathbf{a}, \mathbf{b})-\frac{2 \sqrt{C}}{1-\sqrt{\beta}}\right]^{+} d p(\mathbf{a}, \mathbf{b})
\end{array}
$$

where $[\cdot]^{+}=\max (0, \cdot)$.

This implies in particular that for all $k \geq 0$

$$
\mathbb{E}\left(d_{\mathbf{M}_{k}}\left(\mathbf{a}_{k}, \mathbf{b}_{k}\right)\right) \leq \frac{2 \sqrt{C}}{1-\sqrt{\beta}}+\sqrt{\beta}^{k} \mathbb{E}\left(d_{\mathbf{M}_{0}}\left(\mathbf{a}_{0}, \mathbf{b}_{0}\right)\right)
$$

Proof Let $\mathbf{x}=(\mathbf{a}, \mathbf{b})^{T} \in \mathbb{R}^{2 n}$. We have by the triangle inequality (to avoid long formulas, we drop the second argument of $\mathbf{f}$ and $\sigma$ in the following calculations)

$$
\begin{aligned}
d_{\mathbf{M}_{k+1}}\left(\mathbf{a}_{k+1}, \mathbf{b}_{k+1}\right) & \leq d_{\mathbf{M}_{k+1}}\left(\mathbf{f}\left(\mathbf{a}_{k}\right), \mathbf{f}\left(\mathbf{b}_{k}\right)\right) \\
& +d_{\mathbf{M}_{k+1}}\left(\mathbf{f}\left(\mathbf{a}_{k}\right), \mathbf{f}\left(\mathbf{a}_{k}\right)+\sigma\left(\mathbf{a}_{k}\right) w_{k+1}\right) \\
& +d_{\mathbf{M}_{k+1}}\left(\mathbf{f}\left(\mathbf{b}_{k}\right), \mathbf{f}\left(\mathbf{b}_{k}\right)+\sigma\left(\mathbf{b}_{k}\right) w_{k+1}^{\prime}\right)
\end{aligned}
$$

Let us examine the conditional expectations of the three terms of the right hand side

- From (H1) and lemma 1 one has

$$
\mathbb{E}_{\mathbf{x}}\left(d_{\mathbf{M}_{k+1}}\left(\mathbf{f}\left(\mathbf{a}_{k}\right), \mathbf{f}\left(\mathbf{b}_{k}\right)\right)\right) \leq \sqrt{\beta} \mathbb{E}_{\mathbf{x}}\left(d_{\mathbf{M}_{k}}\left(\mathbf{a}_{k}, \mathbf{b}_{k}\right)\right)
$$

- Next, from (H2)

$$
\mathbb{E}_{\mathbf{x}}\left(d_{\mathbf{M}_{k+1}}\left(\mathbf{f}\left(\mathbf{a}_{k}\right), \mathbf{f}\left(\mathbf{a}_{k}\right)+\sigma\left(\mathbf{a}_{k}\right) w_{k+1}\right)\right) \leq \sqrt{C}
$$

and similarly for $d_{\mathbf{M}_{k+1}}\left(\mathbf{f}\left(\mathbf{b}_{k}\right), \mathbf{f}\left(\mathbf{b}_{k}\right)+\sigma\left(\mathbf{b}_{k}\right) w_{k+1}^{\prime}\right)$.

If we now set $u_{k}=\mathbb{E}_{\mathbf{x}}\left(d_{\mathbf{M}_{k}}\left(\mathbf{a}_{k}, \mathbf{b}_{k}\right)\right)$ then the above implies

$$
u_{k+1} \leq \sqrt{\beta} u_{k}+2 \sqrt{C}
$$

Define next $v_{k}=u_{k}-2 \sqrt{C} /(1-\sqrt{\beta})$. Then replacing $u_{k}$ by $v_{k}+2 \sqrt{C} /(1-\sqrt{\beta})$ in (5) yields

$$
v_{k+1} \leq \sqrt{\beta} v_{k}
$$

This implies that $\forall k \geq 0, v_{k} \leq v_{0} \sqrt{\beta}^{k} \leq\left[v_{0}\right]^{+} \sqrt{\beta}^{k}$. Replacing $v_{k}$ by its expression in terms of $u_{k}$ then yields

$$
\forall k \geq 0 \quad u_{k} \leq \frac{2 \sqrt{C}}{1-\sqrt{\beta}}+\sqrt{\beta}^{k}\left[u_{0}-\frac{2 \sqrt{C}}{1-\sqrt{\beta}}\right]^{+}
$$

which is the desired result.

Next, integrating the last inequality with respect to $\mathrm{x}$ leads to (3). Finally, (4) follows from (3) by remarking that

$$
\begin{gathered}
\int\left[d_{\mathbf{M}_{0}}(\mathbf{a}, \mathbf{b})-\frac{\sqrt{C}}{1-\sqrt{\beta}}\right]^{+} d p(\mathbf{a}, \mathbf{b}) \leq \\
\int d_{\mathbf{M}_{0}}(\mathbf{a}, \mathbf{b}) d p(\mathbf{a}, \mathbf{b})=\mathbb{E}\left(d_{\mathbf{M}_{0}}(\mathbf{a}, \mathbf{b})\right)
\end{gathered}
$$

Remark In the particular context of state-independent metrics, hypothesis (H2) is equivalent to the following simpler condition

$$
\forall \mathbf{a}, k \quad \operatorname{tr}\left(\sigma(\mathbf{a}, k)^{T} \mathbf{M}_{k+1} \sigma(\mathbf{a}, k) \mathbf{Q}_{k}\right) \leq C
$$

Also, for state-independent metrics, one has

$$
d_{\mathbf{M}_{k}}\left(\mathbf{a}_{k}, \mathbf{b}_{k}\right)^{2}=\left\|\mathbf{a}_{k}-\mathbf{b}_{k}\right\|_{\mathbf{M}_{k}}^{2}=\left(\mathbf{a}_{k}-\mathbf{b}_{k}\right)^{T} \mathbf{M}_{k}\left(\mathbf{a}_{k}-\mathbf{b}_{k}\right)
$$

which leads to the following stronger result instead of (4)

$$
\begin{gathered}
\mathbb{E}\left(\left\|\mathbf{a}_{k}-\mathbf{b}_{k}\right\|_{\mathbf{M}_{k}}^{2}\right) \leq \frac{2 C}{1-\beta}+\beta^{k} \mathbb{E}\left(\left\|\mathbf{a}_{0}-\mathbf{b}_{0}\right\|_{\mathbf{M}_{0}}^{2}\right) \\
\text { III. HYBRID SYSTEMS }
\end{gathered}
$$

We have derived above the discrete stochastic contraction theorem for time- and state-dependent metrics, contrary to the context of continuous systems, where the state-dependentmetrics version of the contraction theorem is still unproved [4]. 
We now address the case of hybrid systems, but due to the current limitations of continuous stochastic contraction, only state-independent metrics will be considered.

For clarity, we assume in this paper constant dwell-times, although more elaborate conditions regarding dwell-times can be adapted from [3].

Consider the hybrid resetting stochastic dynamical system

$$
\begin{aligned}
& \forall k \geq 0 \quad \mathbf{a}\left(k \tau^{+}\right)=\mathbf{f}_{d}\left(\mathbf{a}\left(k \tau^{-}\right), k\right)+\sigma_{d}\left(\mathbf{a}\left(k \tau^{-}\right), k\right) w_{k} \\
& \forall k \geq 0, \quad \forall t \in] k \tau,(k+1) \tau\left[\quad d \mathbf{a}=\mathbf{f}_{c}(\mathbf{a}, t) d t+\sigma_{c}(\mathbf{a}, t) d W\right.
\end{aligned}
$$

All the contraction properties below will be stated with respect to a uniformly positive definite time-varying metric $\mathbf{M}(t)=\mathbf{\Theta}(t)^{T} \mathbf{\Theta}(t)$. Furthermore, it will be assumed that for all $k \geq 0, \mathbf{M}$ is continuously differentiable in $] k \tau,(k+1) \tau[$. Finally, $\mathbf{M}\left(k \tau^{-}\right)$and $\mathbf{M}\left(k \tau^{+}\right)$will respectively denote the left and right limits of $\mathbf{M}(t)$ at $t=k \tau$ (and similarly for $\mathbf{\Theta}$ ).

\section{A. The discrete and continuous parts are both contracting}

Theorem 2 (Hybrid stochastic contraction): Assume the following conditions

(i) For all $k$, the discrete part is stochastically contracting at $k \tau$ with rate $\beta<1$ and bound $C_{d}$, i.e.

$$
\forall \mathbf{a} \in \mathbb{R}^{n} \quad \lambda_{\max }\left(\mathbf{F}(k \tau)^{T} \mathbf{F}(k \tau)\right) \leq \beta
$$

where $\mathbf{F}(k \tau)=\mathbf{\Theta}\left(k \tau^{+}\right) \frac{\partial \mathbf{f}_{d}}{\partial \mathbf{a}}(\mathbf{a}, k) \boldsymbol{\Theta}\left(k \tau^{-}\right)$, and

$$
\forall \mathbf{a} \in \mathbb{R}^{n} \quad \operatorname{tr}\left(\sigma_{d}(\mathbf{a}, k)^{T} \mathbf{M}\left(k \tau^{+}\right) \sigma_{d}(\mathbf{a}, k) \mathbf{Q}_{k}\right) \leq C_{d}
$$

(ii) For all $k$, the continuous part is stochastically contracting in $] k \tau,(k+1) \tau$ [ with rate $\lambda>0$ and bound $C_{c}$, i.e. $\left.\forall \mathbf{a} \in \mathbb{R}^{n}, \forall t \in\right] k \tau,(k+1) \tau[$,

$$
\begin{gathered}
\lambda_{\max }\left(\left(\frac{d}{d t} \boldsymbol{\Theta}(t)+\boldsymbol{\Theta}(t) \frac{\partial \mathbf{f}}{\partial \mathbf{a}}\right) \boldsymbol{\Theta}^{-1}(t)\right)_{s} \leq-\lambda \\
\operatorname{tr}\left(\sigma_{c}(\mathbf{a}, t)^{T} \mathbf{M}(t) \sigma_{c}(\mathbf{a}, t)\right) \leq C_{c}
\end{gathered}
$$

Let $\mathbf{a}(t)$ and $\mathbf{b}(t)$ be two trajectories whose initial conditions are given by a probability distribution $p(\mathbf{x}(0))=$ $p(\mathbf{a}(0), \mathbf{b}(0))$. Then for all $t \geq 0$

$$
\begin{gathered}
\mathbb{E}\left(\|\mathbf{a}(t)-\mathbf{b}(t)\|_{\mathbf{M}(t)}^{2}\right) \leq \\
C_{1}+\mathbb{E}\left(\|\mathbf{a}(0)-\mathbf{b}(0)\|_{\mathbf{M}(0)}^{2}\right) \beta^{\lfloor t / \tau\rfloor} e^{-2 \lambda t}
\end{gathered}
$$

where $C_{1}=\frac{2 \lambda C_{d}+(1-\beta)\left(1+\beta-r_{1}\right) C_{c}}{\lambda(1-\beta)\left(1-r_{1}\right)}$ and $r_{1}=\beta e^{-2 \lambda \tau}$.

Proof For all $t \geq 0$, let $u(t)=\mathbb{E}\left(\|\mathbf{a}(t)-\mathbf{b}(t)\|_{\mathbf{M}(t)}^{2}\right)$ and let us study the evolution of $u(t)$ between $k \tau^{+}$and $(k+1) \tau^{+}$.

Condition (ii) and theorem 2 of [4] yield

$$
u\left((k+1) \tau^{-}\right) \leq \frac{C_{c}}{\lambda}+u\left(k \tau^{+}\right) e^{-2 \lambda \tau}
$$

Next, condition (i) and theorem 1 above yield

$$
u\left((k+1) \tau^{+}\right) \leq \frac{2 C_{d}}{1-\beta}+\beta u\left((k+1) \tau^{-}\right)
$$

Substituting (9) into (10) leads to

$$
\begin{aligned}
u\left((k+1) \tau^{+}\right) & \leq \frac{2 C_{d}}{1-\beta}+\beta\left(\frac{C_{c}}{\lambda}+\beta u\left(k \tau^{+}\right) e^{-2 \lambda \tau}\right) \\
& =\frac{2 C_{d}}{1-\beta}+\frac{\beta C_{c}}{\lambda}+\beta e^{-2 \lambda \tau} u\left(k \tau^{+}\right)
\end{aligned}
$$

Define $D_{1}=\frac{2 C_{d}}{1-\beta}+\frac{\beta C_{c}}{\lambda}$ and $v_{k}=u\left(k \tau^{+}\right)-D_{1} /\left(1-r_{1}\right)$. Then, similarly to the proof of theorem 1, we have $v_{k+1} \leq$ $r_{1} v_{k}$, and then $v_{k} \leq r_{1}^{k}\left[v_{0}\right]^{+}$, which implies

$$
\begin{aligned}
u\left(k \tau^{+}\right) & \leq \frac{D_{1}}{1-r_{1}}+\left[u\left(0^{+}\right)-\frac{D_{1}}{1-r_{1}}\right]^{+} r_{1}^{k} \\
& \leq \frac{D_{1}}{1-r_{1}}+u\left(0^{+}\right) r_{1}^{k}
\end{aligned}
$$

Now, for any $t \geq 0$, choose $k=\lfloor t / \tau\rfloor$. Then

$$
\begin{aligned}
u(t) & \leq \frac{C_{c}}{\lambda}+u\left(k \tau^{+}\right) e^{-2 \lambda(t-k \tau)} \\
& \leq \frac{C_{c}}{\lambda}+\frac{D_{1} e^{-2 \lambda(t-k \tau)}}{1-r_{1}}+u\left(0^{+}\right) \beta^{k} e^{-2 \lambda t} \\
& \leq \frac{C_{c}}{\lambda}+\frac{D_{1}}{1-r_{1}}+u\left(0^{+}\right) \beta^{k} e^{-2 \lambda t}
\end{aligned}
$$

which leads to the desired result after some algebraic manipulations.

\section{B. Only the discrete part is contracting}

Let us examine now the more interesting case when the continuous part is not contracting, more precisely when $\lambda \leq 0$ in (8). For this, we shall need to revisit the proof of theorem 2 in [4].

Theorem 3 (Case $\lambda=0$ ): Assume all the hypotheses of theorem 2 except that $\lambda=0$ in (8). Then for all $t \geq 0$

$$
\begin{gathered}
\mathbb{E}\left(\|\mathbf{a}(t)-\mathbf{b}(t)\|_{\mathbf{M}(t)}^{2}\right) \leq \\
C_{2}+\mathbb{E}\left(\|\mathbf{a}(0)-\mathbf{b}(0)\|_{\mathbf{M}(0)}^{2}\right) \beta^{\lfloor t / \tau\rfloor}
\end{gathered}
$$

where $C_{2}=\frac{2 C_{d}+2 \beta(1-\beta) C_{c} \tau}{(1-\beta)^{2}}$.

Proof As in the proof of theorem 2 in [4], let

$$
V(\mathbf{x}, t)=V\left((\mathbf{a}, \mathbf{b})^{T}, t\right)=(\mathbf{a}-\mathbf{b})^{T} \mathbf{M}(t)(\mathbf{a}-\mathbf{b})
$$

Lemma 1 of [4] is unchanged, yielding (see [4] for more details)

$$
\forall t \in] k \tau,(k+1) \tau\left[\quad \widetilde{A} V(\mathbf{x}(t), t) \leq 2 C_{c}\right.
$$

where $\widetilde{A}$ is the infinitesimal operator associated with the process $\mathbf{x}(t)$ (see section 2.1.2 of [4] or p. 15 of [10] for more details).

By Dynkin's formula [10], one then obtains for all $\mathbf{x} \in \mathbb{R}^{2 n}$

$$
\begin{aligned}
\mathbb{E}_{\mathbf{x}} V(\mathbf{x}(t), t)-V\left(\mathbf{x}, k \tau^{+}\right) & =\mathbb{E}_{\mathbf{x}} \int_{k \tau}^{t} \widetilde{A} V(\mathbf{x}(s), s) d s \\
& \leq \mathbb{E}_{\mathbf{x}} \int_{k \tau}^{t} 2 C_{c} d s \\
& =2 C_{c}(t-k \tau)
\end{aligned}
$$

Integrating the above inequality with respect to $\mathrm{x}$ then yields

$$
\forall t \in] k \tau,(k+1) \tau\left[\quad u(t) \leq 2 C_{c}(t-k \tau)+u\left(k \tau^{+}\right)\right.
$$

In particular, (9) becomes

$$
u\left((k+1) \tau^{-}\right) \leq 2 C_{c} \tau+u\left(k \tau^{+}\right)
$$


which leads to, after substition into [10),

$$
u\left((k+1) \tau^{+}\right) \leq \frac{2 C_{d}}{1-\beta}+2 \beta C_{c} \tau+\beta u\left(k \tau^{+}\right)
$$

This finally implies

$$
u\left(k \tau^{+}\right) \leq \frac{\frac{2 C_{d}}{1-\beta}+2 \beta C_{c} \tau}{1-\beta}+u\left(0^{+}\right) \beta^{k}
$$

The remainder of the proof can be adapted from that of theorem 2,

Theorem 4 (Case $\lambda<0$ ): Assume all the hypotheses of theorem 2 except that $\lambda<0$ in (8). Let $k=\lfloor t / \tau\rfloor$. There are two cases:

- If $\beta<e^{-2|\lambda| \tau}$, then let $r_{2}=\beta e^{2|\lambda| \tau}<1$. For all $t \geq 0$

$$
\begin{gathered}
\mathbb{E}\left(\|\mathbf{a}(t)-\mathbf{b}(t)\|_{\mathbf{M}(t)}^{2}\right) \leq \\
C_{3}+\mathbb{E}\left(\|\mathbf{a}(0)-\mathbf{b}(0)\|_{\mathbf{M}(0)}^{2}\right) e^{2|\lambda| \tau} r_{2}^{k}
\end{gathered}
$$

where $C_{3}=\frac{2|\lambda| C_{d}+(1-\beta)\left(1+\beta-r_{2}\right) e^{2|\lambda| \tau} C_{c}}{|\lambda|(1-\beta)\left(1-r_{2}\right)}$.

- If $\beta \geq e^{-2|\lambda| \tau}$, then there is - in general - no finite bound on $\mathbb{E}\left(\|\mathbf{a}(t)-\mathbf{b}(t)\|_{\mathbf{M}(t)}^{2}\right)$ as $t \rightarrow+\infty$.

Proof One has now for all $t \in] k \tau,(k+1) \tau[$,

$$
\widetilde{A} V(\mathbf{x}(t), t) \leq 2|\lambda| V(\mathbf{x}(t), t)+2 C_{c}
$$

with $|\lambda|>0$. By Dynkin's formula, one has, for all $\mathbf{x} \in \mathbb{R}^{2 n}$

$\mathbb{E}_{\mathbf{x}} V(\mathbf{x}(t), t)-V\left(\mathbf{x}, k \tau^{+}\right) \leq \mathbb{E}_{\mathbf{x}} \int_{k \tau}^{t}\left(2|\lambda| V(\mathbf{x}(s), s)+2 C_{c}\right) d s$

Let now $g(t)=\mathbb{E}_{\mathbf{x}} V(\mathbf{x}(t), t)$. The above equation then yields

$$
g(t)=V\left(\mathbf{x}, k \tau^{+}\right)+2 C_{c}(t-k \tau)+2|\lambda| \int_{k \tau}^{t} g(s) d s
$$

Applying the classical Gronwall's lemma [11] to $g(t)$ leads to

$$
\begin{aligned}
g(t) \leq & V\left(\mathbf{x}, k \tau^{+}\right)+2 C_{c}(t-k \tau)+ \\
& 2|\lambda| \int_{k \tau}^{t}\left(V\left(\mathbf{x}, k \tau^{+}\right)+2 C_{c} s\right) \exp \left(\int_{s}^{t} 2|\lambda| d u\right) d s \\
= & \frac{C_{c}}{|\lambda|}\left(e^{2|\lambda|(t-k \tau)}-1\right)+V\left(\mathbf{x}, k \tau^{+}\right) e^{2|\lambda|(t-k \tau)}
\end{aligned}
$$

Integrating the above inequality with respect to $\mathrm{x}$ then yields $\forall t \in] k \tau,(k+1) \tau[$,

$$
u(t) \leq \frac{C_{c}}{|\lambda|}\left(e^{2|\lambda|(t-k \tau)}-1\right)+u\left(k \tau^{+}\right) e^{2|\lambda|(t-k \tau)}
$$

which implies

$$
u\left((k+1) \tau^{+}\right) \leq D_{2}+\beta e^{2|\lambda| \tau} u\left(k \tau^{+}\right)
$$

where $D_{2}=\frac{2 C_{d}}{1-\beta}+\frac{\beta C_{c}}{|\lambda|}\left(e^{2|\lambda| \tau}-1\right)$.

There are three cases:

- If $\beta<e^{-2|\lambda| \tau}$, then $r_{2}=\beta e^{2|\lambda| \tau}<1$. By the same reasoning as in theorem 1 one obtains

$$
u\left(k \tau^{+}\right) \leq \frac{D_{2}}{1-r_{2}}+u\left(0^{+}\right) r_{2}^{k}
$$

The remainder of the proof can be adapted from that of theorem 2
- If $\beta=e^{-2|\lambda| \tau}$, then (11) reads

$$
u\left((k+1) \tau^{+}\right) \leq D_{2}+u\left(k \tau^{+}\right)
$$

which implies $\forall k \geq 0, u\left(k \tau^{+}\right) \leq k D_{2}+u\left(0^{+}\right)$. From this, it is clear that there is - in general - no finite bound for $u\left(k \tau^{+}\right)$.

- If $\beta>e^{-2|\lambda| \tau}$, then $r_{2}=\beta e^{2|\lambda| \tau}>1$. By the same reasoning as in theorem 1 , one obtains

$$
u\left(k \tau^{+}\right) \leq\left(u\left(0^{+}\right)+\frac{D_{2}}{r_{2}-1}\right) r_{2}^{k}-\frac{D_{2}}{r_{2}-1}
$$

Since $r_{2}>1$ in this case, it is clear that there is - in general - no finite bound for $u\left(k \tau^{+}\right)$.

Remarks Theorems 3 and 4 show that it is possible to stabilize an unstable system by discrete resettings. If the continuous system is indifferent $(\lambda=0)$, then any sequence of uniformly contracting resettings is stabilizing. However, it should be noted that the asymptotic bound $C_{2} \rightarrow \infty$ when $\beta \rightarrow 1$. In contrast, if the continuous system is strictly unstable $(\lambda<0)$, then specific contraction rates (depending on the dwell-time and the "expansion" rate of the continuous system) of the resettings are required. Finally, note that in both cases, the asymptotic bounds $C_{2}$ and $C_{3}$ are increasing functions of the dwell-time $\tau$.

\section{Comments}

\section{A. Modelling issue: distinct driving noise}

In the same spirit as [4], and contrary to previous works on the stability of stochastic systems [12], the $\mathbf{a}$ and $\mathbf{b}$ systems considered in sections $\Pi$ and $\Pi$ are driven by distinct and independent noise processes. This approach enables us to study the stability of the system with respect to variations in initial conditions and to random perturbations: indeed, two trajectories of any real-life system are typically affected by distinct realizations of the noise. In addition, this approach leads very naturally to nice results regarding the comparison of noisy and noise-free trajectories (see section [V-B], which are particularly useful in applications (see e.g. section $\mathbf{V}$.

However, because of the very fact that the two trajectories are driven by distinct noise processes, we cannot expect the influence of noise to vanish when the two trajectories get very close to each other. As a consequence, the asymptotic bounds $2 C /(1-\beta)$ (for discrete systems) and $C_{1}, C_{2}, C_{3}$ (for hybrid systems) are strictly positive. These bounds are nevertheless optimal, in the sense that they can be attained (adapt the Ornstein-Uhlenbeck example in section 2.3.1 of [4]).

\section{B. Noisy and noise-free trajectories}

Instead of considering two noisy trajectories $\mathbf{a}$ and $\mathbf{b}$ as in theorem 1, we assume now that $\mathbf{a}$ is noisy, while $\mathbf{b}$ is noisefree. More precisely, for all $k \in \mathbb{N}$

$$
\begin{gathered}
\mathbf{a}_{k+1}=\mathbf{f}\left(\mathbf{a}_{k}, k\right)+\sigma\left(\mathbf{a}_{k}, k\right) w_{k+1} \\
\mathbf{b}_{k+1}=\mathbf{f}\left(\mathbf{b}_{k}, k\right)
\end{gathered}
$$


To show the exponential convergence of $\mathbf{a}$ and $\mathbf{b}$ to each other, one can follow the same reasoning as in the proof of theorem [1] with $C$ is replaced by $C / 2$. This leads to the following result

Corollary 1: Assume all the hypothesis of theorem 1 and consider a noise-free trajectory $\mathbf{b}_{k}$ and a noisy trajectory $\mathbf{a}_{k}$ whose initial conditions are given by a probability distribution $p\left(\mathbf{a}_{0}\right)$. Then, for all $k \in \mathbb{N}$

$$
\begin{array}{r}
\mathbb{E}\left(\left\|\mathbf{a}_{k}-\mathbf{b}_{k}\right\|_{\mathbf{M}_{k}}^{2}\right) \leq \frac{C}{1-\beta}+ \\
\beta^{k} \int\left[\left\|\mathbf{a}-\mathbf{b}_{0}\right\|_{\mathbf{M}_{0}}^{2}-\frac{C}{1-\beta}\right]^{+} d p(\mathbf{a})
\end{array}
$$

Remarks

- The above derivation of corollary 1 is only permitted by our choice of considering distinct driving noise processes for systems $\mathbf{a}$ and $\mathbf{b}$ (see section IV-A).

- Based on theorems 2, 3 and 4, similar corollaries can be obtained for hybrid systems.

- These corollaries provide a robustness result for contracting discrete and hybrid systems, in the sense that any contracting system is automatically protected against noise, as quantified by (12). This robustness could be related to the exponential nature of contraction stability.

\section{APPLICATION: OSCILLATOR SYNCHRONIZATION BY DISCRETE COUPLINGS}

Using the above developped tools, we study in this section the synchronization of nonlinear oscillators in presence of random perturbations. The novelty here is that the interactions between the oscillators occur at discrete time instants, contrary to many previous works devoted to synchronization in the state-space1 [14], [7].

Specifically, consider the Central Pattern Generator (CPG) delivering $2 \pi / 3$-phase-locked signals of section 5.3 in [7]. This CPG consists of a network of three Andronov-Hopf oscillators $\mathbf{x}_{i}=\left(x_{i}, y_{i}\right)^{T}, i=1,2,3$. We construct below a discretecouplings version of this CPG.

At instants $t=k \tau, k \in \mathbb{N}$, the three oscillators are coupled in the following way (assuming noisy measurements)

$$
\begin{aligned}
\mathbf{x}_{i}\left(k \tau^{+}\right) & =\mathbf{x}_{i}\left(k \tau^{-}\right) \\
& +\gamma\left(\mathbf{R}\left(\mathbf{x}_{i+1}\left(k \tau^{-}\right)+\frac{\sigma_{d}}{\sqrt{2}} w_{k}\right)-\mathbf{x}_{i}\left(k \tau^{-}\right)\right)
\end{aligned}
$$

with $\mathbf{x}_{4}=\mathbf{x}_{1}$ and

$$
\mathbf{R}=\left(\begin{array}{cc}
-\frac{1}{2} & -\frac{\sqrt{3}}{2} \\
\frac{\sqrt{3}}{2} & -\frac{1}{2}
\end{array}\right)
$$

Between two interaction instants, the oscillators follow the uncoupled, noisy, dynamics

$$
d \mathbf{x}_{i}=\mathbf{f}\left(\mathbf{x}_{i}\right) d t+\frac{\sigma_{c}}{\sqrt{2}} d W
$$

\footnotetext{
${ }^{1}$ Discrete couplings are more frequent in the literature devoted to phase oscillators synchronization, where phase reduction techniques are used [13]. However, contrary to our approach, these techniques are only applicable in the case of weak coupling strenghs and small noise intensities.
}

where

$$
\mathbf{f}\left(\mathbf{x}_{i}\right)=\mathbf{f}\left(\begin{array}{c}
x_{i} \\
y_{i}
\end{array}\right)=\left(\begin{array}{c}
x_{i}-y_{i}-x_{i}^{3}-x_{i} y_{i}^{2} \\
x_{i}+y_{i}-y_{i}^{3}-y_{i} x_{i}^{2}
\end{array}\right)
$$

We apply now the projection technique developped in [7], [4]. We recommend the reader to refer to these papers for more details about the following calculations.

Consider first the (linear) subspace $\mathcal{M}$ of the global state space (the global state is defined by $\widehat{\mathbf{x}}=\left(\mathbf{x}_{1}, \mathbf{x}_{2}, \mathbf{x}_{3}\right)^{T}$ ) where the oscillators are $2 \pi / 3$-phase-locked

$$
\mathcal{M}=\left\{\left(\mathbf{R}^{2}(\mathbf{x}), \mathbf{R}(\mathbf{x}), \mathbf{x}\right)^{T}: \mathbf{x} \in \mathbb{R}^{2}\right\}
$$

Let $\mathbf{V}$ and $\mathbf{U}$ be two orthonormal projections on $\mathcal{M}^{\perp}$ and $\mathcal{M}$ respectively and consider $\widehat{\mathbf{y}}=\mathbf{V} \widehat{\mathbf{x}}$. Since the mapping is linear, using Itô differentiation rule yields the following dynamics for $\widehat{\mathbf{y}}$

$$
\begin{aligned}
& \forall k \in \mathbb{N} \quad \widehat{\mathbf{y}}\left(k \tau^{+}\right)=\mathbf{g}_{d}\left(\widehat{\mathbf{y}}\left(k \tau^{-}\right)\right)+\gamma \frac{\sigma_{d}}{\sqrt{2}} w_{k} \\
& \forall t \in] k \tau,(k+1) \tau\left[\quad d \widehat{\mathbf{y}}=\mathbf{g}_{c}(\widehat{\mathbf{y}}) d t+\frac{\sigma_{c}}{\sqrt{2}} d W\right.
\end{aligned}
$$

with

$$
\begin{gathered}
\mathbf{g}_{d}(\widehat{\mathbf{y}})=\mathbf{V L x}=\mathbf{V L}\left(\mathbf{V}^{T} \widehat{\mathbf{y}}+\mathbf{U}^{T} \mathbf{U} \widehat{\mathbf{x}}\right)=\mathbf{V L V}^{T} \widehat{\mathbf{y}} \\
\mathbf{g}_{c}(\widehat{\mathbf{y}})=\mathbf{V} \widehat{\mathbf{f}}\left(\mathbf{V}^{T} \widehat{\mathbf{y}}+\mathbf{U}^{T} \mathbf{U} \widehat{\mathbf{x}}\right)
\end{gathered}
$$

where

$$
\begin{gathered}
\mathbf{L}=\left(\begin{array}{ccc}
(1-\gamma) \mathbf{I}_{2} & \gamma \mathbf{R} & \mathbf{0} \\
\mathbf{0} & (1-\gamma) \mathbf{I}_{2} & \gamma \mathbf{R} \\
\gamma \mathbf{R} & \mathbf{0} & (1-\gamma) \mathbf{I}_{2}
\end{array}\right) \\
\hat{\mathbf{f}}(\widehat{\mathbf{x}})=\left(\mathbf{f}\left(\mathbf{x}_{1}\right), \mathbf{f}\left(\mathbf{x}_{2}\right), \mathbf{f}\left(\mathbf{x}_{3}\right)\right)^{T}
\end{gathered}
$$

Remark that $\mathbf{g}_{d}(\mathbf{0})=\mathbf{0}$ and $\mathbf{g}_{c}(\mathbf{0})=\mathbf{0}$ (the last equality holds because of the symmetry of $\mathbf{f}: \forall \mathbf{x}, \mathbf{f}(\mathbf{R x})=\mathbf{R}(\mathbf{f}(\mathbf{x}))$ ). Thus, $\mathbf{0}$ is a particular solution to the noise-free version of the hybrid stochastic system (13]14).

Let us now examine the contraction properties of equations (13) and (14).

We have first

$$
\frac{\partial \mathbf{g}_{d}{ }^{T}}{\partial \widehat{\mathbf{y}}} \frac{\partial \mathbf{g}_{d}}{\partial \widehat{\mathbf{y}}}=\mathbf{V} \mathbf{L}^{T} \mathbf{V}^{T} \mathbf{V} \mathbf{L} \mathbf{V}^{T}=\left(3 \gamma^{2}-3 \gamma+1\right) \mathbf{I}_{4}
$$

so that $\lambda_{\max }\left(\frac{\partial \mathbf{g}_{d}}{\partial \mathbf{y}}{ }^{T} \frac{\partial \mathbf{g}_{d}}{\partial \mathbf{y}}\right)=3 \gamma^{2}-3 \gamma+1<1$ (for $0<\gamma<1$ ).

Second,

$$
\frac{\partial \mathbf{g}_{c}}{\partial \widehat{\mathbf{y}}}=\mathbf{V} \frac{\partial \hat{\mathbf{f}}}{\partial \widehat{\mathbf{x}}} \mathbf{V}^{T}=\mathbf{V}\left(\begin{array}{ccc}
\frac{\partial \mathbf{f}}{\partial \mathbf{x}}\left(\mathbf{x}_{1}\right) & \mathbf{0} & \mathbf{0} \\
\mathbf{0} & \frac{\partial \mathbf{f}}{\partial \mathbf{x}}\left(\mathbf{x}_{2}\right) & \mathbf{0} \\
\mathbf{0} & \mathbf{0} & \frac{\partial \mathbf{f}}{\partial \mathbf{x}}\left(\mathbf{x}_{3}\right)
\end{array}\right) \mathbf{V}^{T}
$$

Now observe that $\lambda_{\max }\left(\frac{\partial \mathbf{f}}{\partial \mathbf{x}}\right)_{s}=1-x^{2}-y^{2} \leq 1$. Since $\mathbf{V}$ is an orthonormal projection, one then has $\lambda_{\max }\left(\frac{\partial \mathbf{g}_{c}}{\partial \mathbf{y}}\right)_{s} \leq 1$.

Therefore, if

$$
3 \gamma^{2}-3 \gamma+1<e^{-2 \tau}
$$

then theorem 4 together with the corollaries of section IV-B 
imply that, after exponential transients,

$$
\mathbb{E}\left(\|\widehat{\mathbf{y}}\|^{2}\right) \leq \frac{2 \gamma^{2} \sigma_{d}^{2}+(1-\beta)\left(1+\beta-\beta e^{2 \tau}\right) e^{2 \tau} \sigma_{c}^{2}}{2(1-\beta)\left(1-\beta e^{2 \tau}\right)}
$$

where $\beta=3 \gamma^{2}-3 \gamma+1$.

To conclude, observe that

$$
\|\widehat{\mathbf{y}}\|^{2}=\|\mathbf{V} \widehat{\mathbf{x}}\|^{2}=\frac{1}{3} \sum_{i=1}^{3}\left\|\mathbf{R} \mathbf{x}_{i+1}-\mathbf{x}_{i}\right\|^{2}
$$

Define the phase-locking quality $\delta$ by

$$
\delta=\sum_{i=1}^{3}\left\|\mathbf{R} \mathbf{x}_{i+1}-\mathbf{x}_{i}\right\|^{2}
$$

then one finally obtains

$$
\mathbb{E}(\delta) \leq \frac{6 \gamma^{2} \sigma_{d}^{2}+3(1-\beta)\left(1+\beta-\beta e^{2 \tau}\right) e^{2 \tau} \sigma_{c}^{2}}{2(1-\beta)\left(1-\beta e^{2 \tau}\right)}
$$

after exponential transients.

A numerical simulation is provided in Fig. 1. (a)

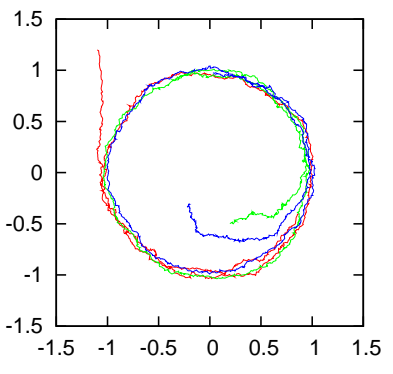

(b)
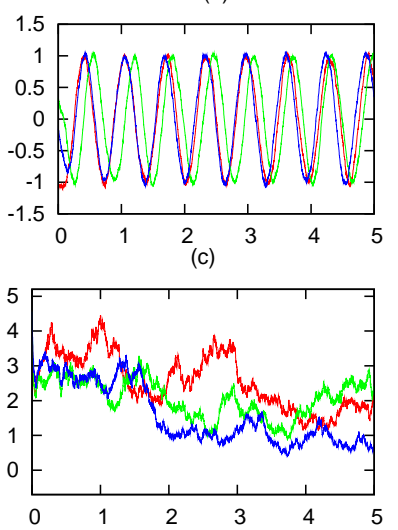

(d)

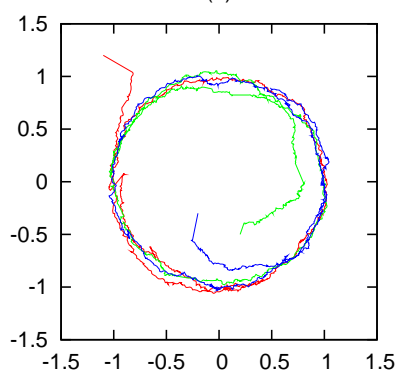

(e)
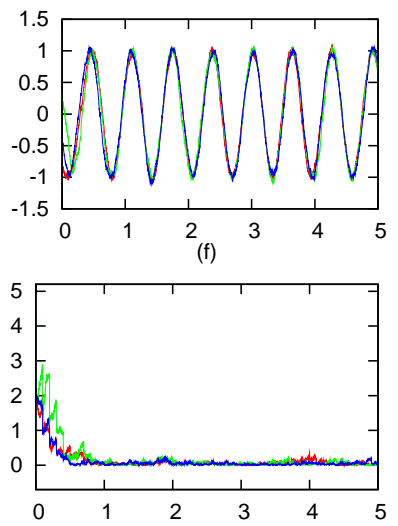

Fig. 1. Numerical simulation using the Euler-Maruyama algorithm [15]. The following set of parameters was used: $\sigma_{c}=0.1, \sigma_{d}=0.05, \tau=0.1$. Two coupling strengths were tested: $\gamma_{\text {weak }}=0.01$ for plots (a), (b), (c), and $\gamma_{\text {strong }}=0.2$ for plots (c), (d), (e). Note that $\gamma_{\text {weak }}$ does not satisfy

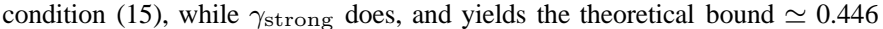
(as provided by 16 ) on the phase-locking quality $\delta$. Plots (a) and (d) show the $2 \mathrm{~d}$ trace of sample trajectories of the three oscillators for $t \in[0,1]$. Plots (b) and (e) show sample trajectories of the first coordinates of $\mathbf{x}_{1}, \mathbf{R}\left(\mathbf{x}_{2}\right)$ and $\mathbf{R}^{2}\left(\mathbf{x}_{3}\right)$ as functions of time. Plot (c) and (f) show three sample trajectories of $\delta$.

\section{PERSPECTIVES}

We are now focusing on the following directions of research:

- proving the state-dependent-metrics version of the continuous and hybrid stochastic contraction theorems,

- developping more elaborate conditions on dwell-times, and also hybrid switched versions of the theorems,

- applying the synchronization-by-discrete-couplings analysis to other types of coupled dynamical systems,

- studying the robustness of hybrid controllers and observers against random perturbations (for instance, the discrete observer for inertial navigation developped in [16]).

\section{ACKNOWLEDGMENT}

The author is grateful to Prof J.-J. Slotine and N. Tabareau for stimulating discussions, and to Dr H. Hicheur for the careful reading of the manuscript. This work has been supported by EC - contract number FP6-IST-027140, action line: Cognitive Systems. This publication reflects only the author's views. The European Community is not liable for any use that may be made of the information contained therein.

\section{REFERENCES}

[1] W. Lohmiller and J.-J. Slotine, "On contraction analysis for nonlinear systems," Automatica, vol. 34, pp. 671-682, 1998.

[2] W. Wang and J.-J. E. Slotine, "On partial contraction analysis for coupled nonlinear oscillators." Biol Cybern, vol. 92, no. 1, pp. 38-53, Jan. 2005.

[3] K. El Rifai and J.-J. Slotine, "Compositional contraction analysis of resetting hybrid systems," IEEE Transactions on Automatic Control, vol. 51, no. 9, pp. 1536-1541, 2006.

[4] Q.-C. Pham, N. Tabareau, and J.-J. Slotine. (2007) A contraction theory approach to stochastic incremental stability. [Online]. Available: http://arxiv.org/abs/0704.0926

[5] W. Lohmiller and J. Slotine, "Control system design for mechanical systems using contraction theory," IEEE Transactions on Automatic Control, vol. 45, no. 5, pp. 984-989, 2000.

[6] W. Lohmiller and J.-J. Slotine, "Nonlinear process control using contraction theory," A.I.Ch.E. Journal, 2000.

[7] Q.-C. Pham and J.-J. Slotine, "Stable concurrent synchronization in dynamic system networks." Neural Netw, vol. 20, no. 1, pp. 62-77, Jan. 2007.

[8] B. Girard, N. Tabareau, Q.-C. Pham, A. Berthoz, and J.-J. Slotine, "Where neuroscience and dynamic system theory meet autonomous robotics: a contracting basal ganglia model for action selection," Neural Networks, 2008.

[9] N. Aghannan and P. Rouchon, "An intrinsic observer for a class of lagrangian systems," IEEE Transactions on Automatic Control, vol. 48, 2003.

[10] H. Kushner, Stochastic Stability and Control. Academic Press, 1967.

[11] J. Robbin. (2006) Gronwall's inequality. [Online]. Available: www.math.wisc.edu/\$ $\backslash$ sim\$robbin/angelic/gronwall.pdf

[12] P. Florchinger, "Lyapunov-like techniques for stochastic stability," SIAM Journal of Control and Optimization, vol. 33, pp. 1151-1169, 1995.

[13] E. Izhikevich, "Weakly pulse-coupled oscillators, FM interactions, synchronization, and oscillatory associative memory," Neural Networks, IEEE Transactions on, vol. 10, no. 3, pp. 508-526, 1999.

[14] A. Pogromsky, G. Santoboni, and H. Nijmeijer, "Partial synchronization: from symmetry towards stability," Physica D: Nonlinear Phenomena, vol. 172 , no. $1-4$, pp. $65-87,2002$.

[15] D. Higham, "An algorithmic introduction to numerical simulation of stochastic differential equations," SIAM Review, vol. 43, pp. 525-546, 2001.

[16] Y. Zhao and J.-J. Slotine, "Discrete nonlinear observers for inertial navigation," Systems and Control Letters, vol. 54, 2005. 